JINOTEP Vol 8 (1) (2021): 68-79

DOI: $10.17977 / \mathrm{um} 031 \mathrm{v} 8 \mathrm{i} 12021 \mathrm{p} 068$

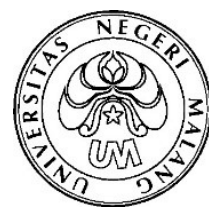

JINOTEP (Jurnal Inovasi Teknologi Pembelajaran)

Kajian dan Riset Dalam Teknologi Pembelajaran

http://journal2.um.ac.id/index.php/jinotep/index

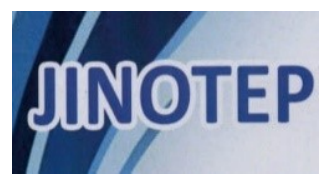

\title{
THE FUTURE OF NEW LEARNING WAY FOR THE VISUALLY IMPAIRED LEARNERS: USE BIONIC EYE WITH VOICE ASSISTANT
}

\author{
Kao Sheng Che \\ Dept. Communication Engineering National Tsing Hua University-Taiwan
}

\begin{abstract}
Article History
Received: 27-01-2021

Accepted: 11-02-2021

Published: 01-03-2021

Available online: 11-022021

\section{Keywords}

Bionic Eye, Voice

Assistant, The Future of

Abstract

Chatbots can be defined as artificial narrow intelligence (ANI) which can perform a single task such as answering people's question by harnessing the power of machine learning. There is some educational-related chatbots developed to interact with learners in mobile instant messaging (MIM) apps. Nowadays, there are more and more voice assistant developed in our world. They aim to fulfill user requests by choosing the best intent from multiple options generated by its Automated Speech Recognition and Natural Language Understanding sub-systems. Bionic eye is also still develop for the blind, it plays a vital part by restoring the vision. Technologies that are involved in bionic eyes are-multiple unit artificial retina chip system (MARC). This report describes an approach for the design and development of a voice assistant based on chatbot development basis combined with bionic eye application for an eLearning system with the learner's personal needs.
\end{abstract} new learning way,

Visually impaired

people

Corresponding author :

Adress: 9F., No.39, Ln. 41, Sec. 2, Wunhua 3rd Rd, Linkou Dist,New

2021 Universitas Negeri Malang p-ISSN 2406-8780

Taipei City 244, Taiwan e-ISSN 2654-7953

Instansi: National Tsing Hua University,

E-mail: jerrynthu0602@gapp.nthu.edu.tw,

\section{INTRODUCTION}

World Health Organization has estimated that almost 40 million visually impaired people are across the world. Blindness occurs due to Retinitis Pigmentosa, Macular Degeneration, and other age-related macular degenerations. Retinitis Pigmentosa (RP) is a type of inherited disease which damages the light sensitive rods and cones. Rods and cones are mainly located in the retina (Banerjee, Saba, Rana, \& Chakraborty, 2020). As new technology still become advanced, our goal is mainly focused on bring the blind new learning way. Because chatbots have made inroads into the educational environment with its capability of interacting with students in natural language. The common 
ways of implementing chatbots include chatbot taking the role of teacher to delivery instructional materials (Heller, Proctor, Mah, Jewell, \& Cheung, 2005), and chatbot playing the role as a learning partner to facilitate learners' understanding of the subject matter (Fryer, Ainley, Thompson, Gibson, \& Sherlock, 2017). The theoretical rationales employed in this study is based on the Community of Inquiry (COI) framework (Q. Zhang et al., 2020). The effects of the COI framework the MIM chatbot design were measured by students' self-reported surveys.

As for Voice Assistant, it' must overcome complex problems and hence they typically are formed of a number of components: one that transcribes the user speech (Automated Speech Recognition - ASR), one that understands the transcribed utterances (Natural Language Understanding - NLU), one that makes decisions (Decision Making - DM), and one that produces the output speech (TTS) (R. Anantha, Chappidi, \& Dawoodi, 2021; Raviteja Anantha, Chappidi, \& Dawoodi, 2020). Many VAs have similar with the chatbot (Weber, Ritschel, Lingenfelser, \& André, 2018), our discussion will focused on the how to use successful learning chatbot to apply on the VAs system, and also combined with the bionic eye technology to let the blind use the new way to learn.

The remainder of the report discussion is organized as follow steps. First, discuss with the successful chatbot learning application (Schilling \& Shankar, 2019). Second. describe the VAs architecture and theory, Third.Consider the technology development of bionic eye. Finally, Discussed the feasible evaluation and future of this way.

1. Chatbot-assisted Learning:Chatbot is a dialogic system that can interact with users in natural language. It's unlike a human interaction, chatbots can always offer help when people need help (Garcia Brustenga, Fuertes Alpiste, \& Molas Castells, 2018). Chatbots show the pedagogical value in facilitating learners' knowledge of language (Ruan et al., 2019), and improving learners' willingness to interacte (Ayedoun, Hayashi, \& Seta, 2019).

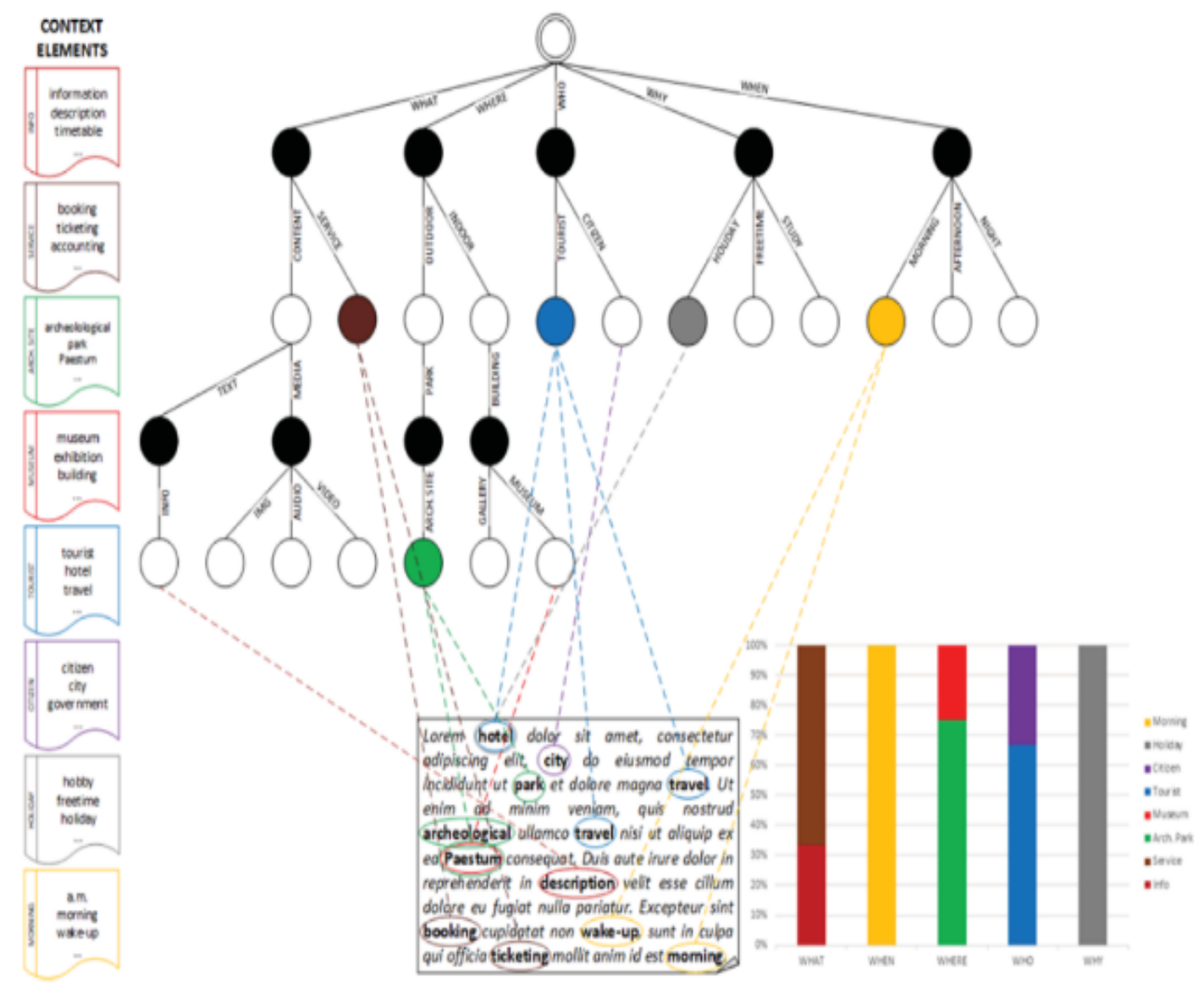

Fig. 1. CHAT: Context Definition (Flora Amato et al., 2019) 
2. The Community of Inquiry as a Theoretical Framework: The Community of Inquiry (COI) framework initially aimed to build a constructivist learning environment in computer conferencing. and it's can also be used in online learning with higher education context (Anderson, Liam, Garrison, \& Archer, 2001). The COI framework is comprised of three ccomponents, which called social presence, cognitive presence, and teaching presence, to declaim the knowledge construction in online learning community (M. Yamada, Goda, Matsukawa, Hata, \& Yasunami, 2016).

3. Latent Dirichlet Allocation: a model can be used to explain the relationship between keywords and topics (in this case, context elements), as shown in the following figure 2. By means of textual analysis, it is possible to know more about the students, it can know where is people or where they would like to be, and the learning purpose of their visit and what they need according to the result (Flora Amato et al., 2019).

4. Voice Assitant Task Description: The final goal of a Voice Assitant is to understand user thinking. The exact meaning of the words is often not enough to choose the best intent. We sorted it into three categories. All privatesensitive information stays on the user's device (R. Anantha et al., 2021).

- Personal Information: e.g. user-location, app subscriptions, browsing history, device-type etc.

- User State: Information about the user's state at the time a query is made.

- Context: Dialog context of what the user said in previous queries in the same conversation or task

5. Bionic Eye Architecture: The main objective of bionic eye is to provide vision to those visually impaired people who are suffering from retinal diseases. By conducting bionic eye in human eye, this way will be able to help a person to recognize the object . In the beginning, a person starts recognizing a tiny flashes in front of them, as the times passes, researchers start learning how to estimate all the tiny flashing lights together and make images by which they can analyze the object.In this process, person can independently do the daily works of life (Banerjee et al., 2020).

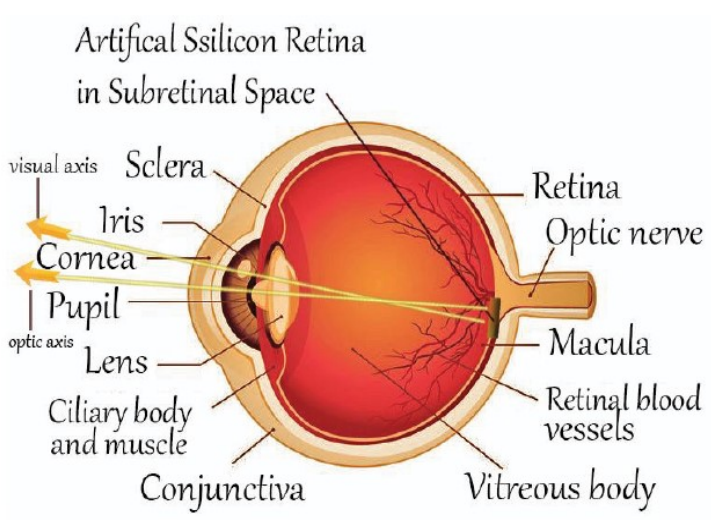

Fig. 2. Bionic eye (Banerjee et al., 2020)

The aim of this review is to analyze the potential of the latest technology for learners experiencing visual problems. This is very important in order to support a learning program for everyone.

\section{METHOD}

We conducted a related review in this study. The methodology was based on general recommendations proposed in related review studies. The methodology included five theory or architecture:

First, we want to design a chatbot as a role of online instructor to teach students from different topic. So we adopt that designing with the COI framework (Yang \&Mohd, 2020). Table 1 presents the information in learning objectives and students' learning materials. Students are expected to interact with the chatbot to recognize the features of image. And Table2 is related to designing examples on Social Presence, Cognitive Presence, and Teaching Presence which will applied on our voice assistant.

Table 1. Summary of Learning Objectives and Learning Materials (Yang \& Mohd, 2020)

\begin{tabular}{ll}
\hline Learning Objectives & Learning Activities \\
\hline $\begin{array}{l}\text { Recognized the } \\
\text { meaning of image }\end{array}$ & $\begin{array}{l}\text { Course introduction } \\
\text { video, discussion on } \\
\text { image from customized } \\
\text { material }\end{array}$ \\
$\begin{array}{l}\text { Video lecture regarding } \\
\text { definition and main } \\
\text { features of image } \\
\text { image definition and } \\
\text { figure out how to } \\
\text { identify image by a } \\
\text { four-step method }\end{array}$ & $\begin{array}{l}\text { An image identification, } \\
\text { video lecture regarding a } \\
\text { four-step method }\end{array}$ \\
$\begin{array}{l}\text { Apply the four step } \\
\text { method to identify } \\
\text { correct image from } \\
\text { cases }\end{array}$ & Case Study \\
\hline
\end{tabular}


Table 2. Design Examples on Social Presence, Cognitive Presence, and Teaching Presence.

\begin{tabular}{|c|c|c|c|}
\hline Element & Categories & Design example & Chatbot prompts example \\
\hline \multirow{3}{*}{$\begin{array}{l}\text { Social } \\
\text { presence }\end{array}$} & Personal/affective & Use emoji & "I'm your learning partner." \\
\hline & $\begin{array}{l}\text { Open } \\
\text { communication }\end{array}$ & Use vocatives & $\begin{array}{l}\text { "Hi Irene, the next video is about the feature } \\
\text { of image." }\end{array}$ \\
\hline & Group cohesion & $\begin{array}{l}\text { Agreements when user gives } \\
\text { right answer }\end{array}$ & "I have the same idea with you." \\
\hline \multirow[t]{4}{*}{$\begin{array}{l}\text { Cognitive } \\
\text { presence }\end{array}$} & Triggering event & $\begin{array}{l}\text { Discussion about an image } \\
\text { example }\end{array}$ & "Do you think this image is correct or false?" \\
\hline & Exploration & $\begin{array}{l}\text { Dialogue regarding the } \\
\text { reason of image }\end{array}$ & "Why do you think it is a true image?" \\
\hline & Integration & $\begin{array}{l}\text { Quizzes to recall the } \\
\text { features of image }\end{array}$ & $\begin{array}{l}\text { "Can you tell me one of the features of } \\
\text { image?" }\end{array}$ \\
\hline & Resolution & Use 4-step method & $\begin{array}{l}\text { "You may need to check the author of this } \\
\text { image }\end{array}$ \\
\hline \multirow[t]{3}{*}{$\begin{array}{l}\text { Teaching } \\
\text { presence }\end{array}$} & $\begin{array}{l}\text { Design and } \\
\text { organization }\end{array}$ & $\begin{array}{l}\text { Illustrate learning outcomes } \\
\text { in intro-video }\end{array}$ & $\begin{array}{l}\text { "Please watch the introduction video to know } \\
\text { your learning objectives." }\end{array}$ \\
\hline & $\begin{array}{l}\text { Facilitating } \\
\text { discourse }\end{array}$ & $\begin{array}{l}\text { Provide step-by-step } \\
\text { instruction }\end{array}$ & $\begin{array}{l}\text { "Now we're going to explore a } 4 \text {-step } \\
\text { method." }\end{array}$ \\
\hline & Direct instruction & Watch instructional videos & $\begin{array}{l}\text { "Please tell me if you finish watch the } \\
\text { video." }\end{array}$ \\
\hline
\end{tabular}

Second, we want to Use LDA Algorithm to discuss with students, the interaction of the user with the chatbot is divided into shorter and simpler sentences (clusters), through appropriate Bayesian filters (Torres-Signes \& Dip, 2020) for keywords, assuming that each sentence is semantically related to the other. Furthermore, using the LDA approach on a set of chats that belong to the same domain, it is possible to automatically extract a Mixed Graph of Terms (mGT) that can be used both for the design of the tree of context and the constraints associated with it is to detect the context extracted in real time from the user's chat with the bot (Flora Amato et al., 2019).
Third, Adopt Bionic MARC System from Bionic Eye The multiple unit artificial retina system composed of a camera, wires, transceiver, chip, stimulation-current driver, silicon rubbers and cables. The camera captures the images which is in front of it and then the images are encoded and transmitted to the transceiver. It is basically a signal which contains encoded data and further rectified and filtered so that the MARC system can be able to extract the data and clock signals, the information fetched are stimulated on the retina (Banerjee et al., 2020). 


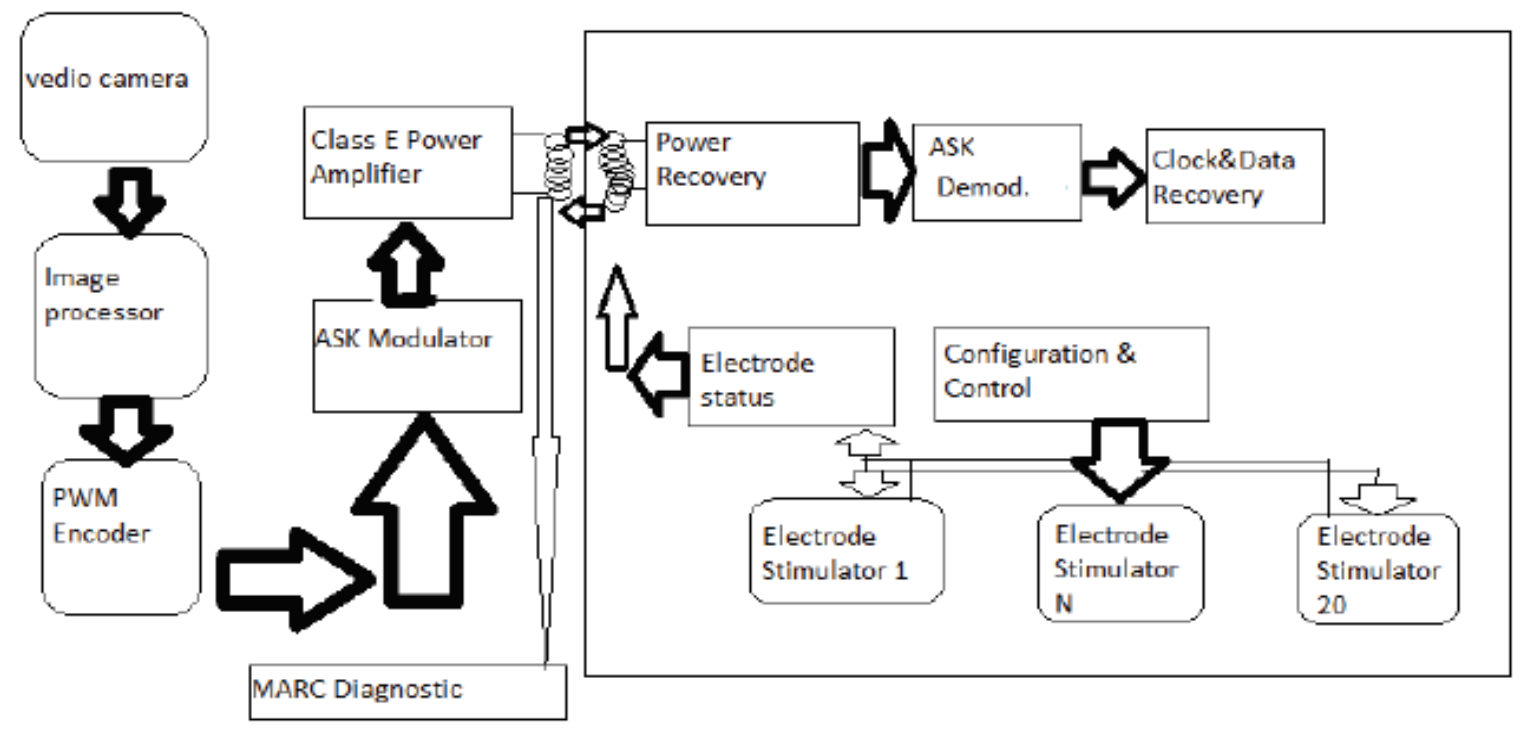

\section{CIRCUIT OF MARC SYSTEM}

Fig. 3. MARC System (Banerjee et al., 2020).

Fourth, develop of our cloud center by method of AWS Lex (Srivastava, Kalra, \& Prabhakar, 2020; Srivastava \& Prabhakar, 2020). To implement this flow course, the trainer should specify characteristics, which are valuable for the course understanding, and provide a few versions of the learning content group based on the characteristics values. The set of characteristics, specified for the course, are stored in DynamoDB, and the educational materials are placed in Amazon S3. The search of the required content is implemented using tagging. The VAs has been designed using the following Amazon services (Davies, Verovko, Verovko, \& Solomakha, 2021).

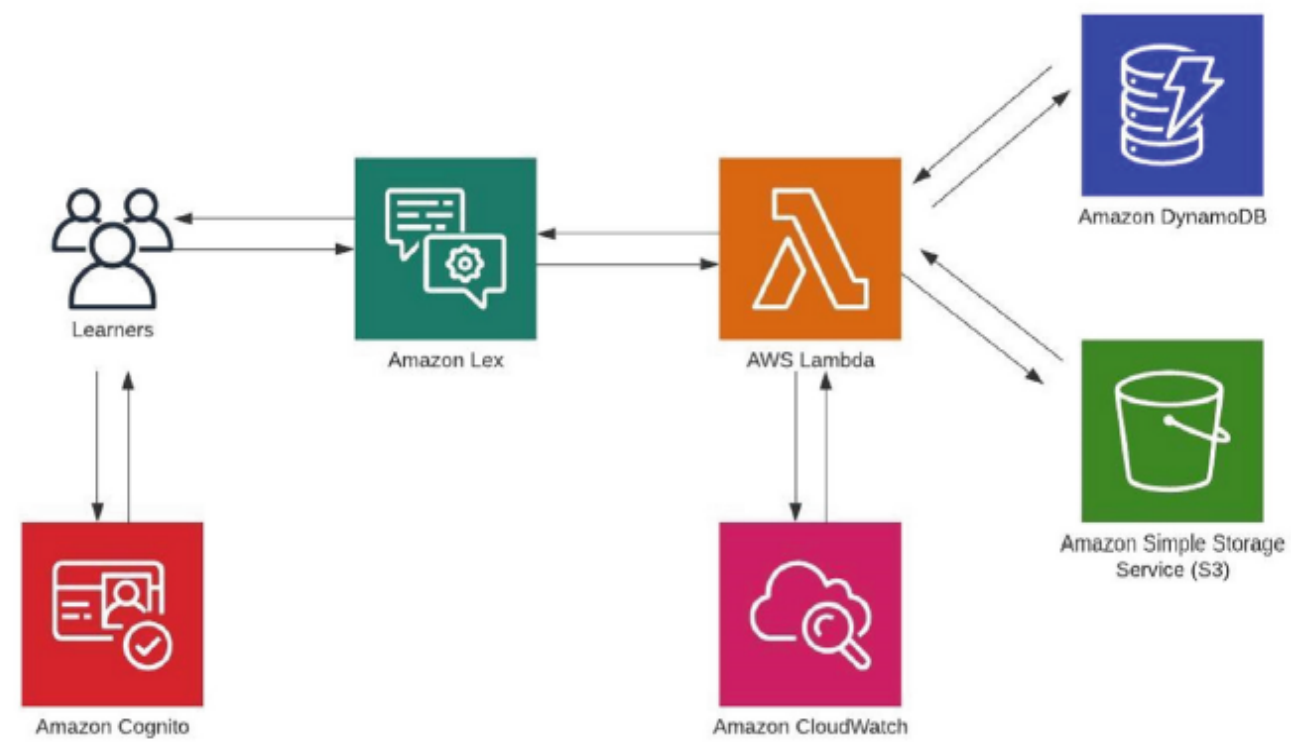

Fig. 4. Voice Assistant Cloud Architecture (Davies et al., 2021) 


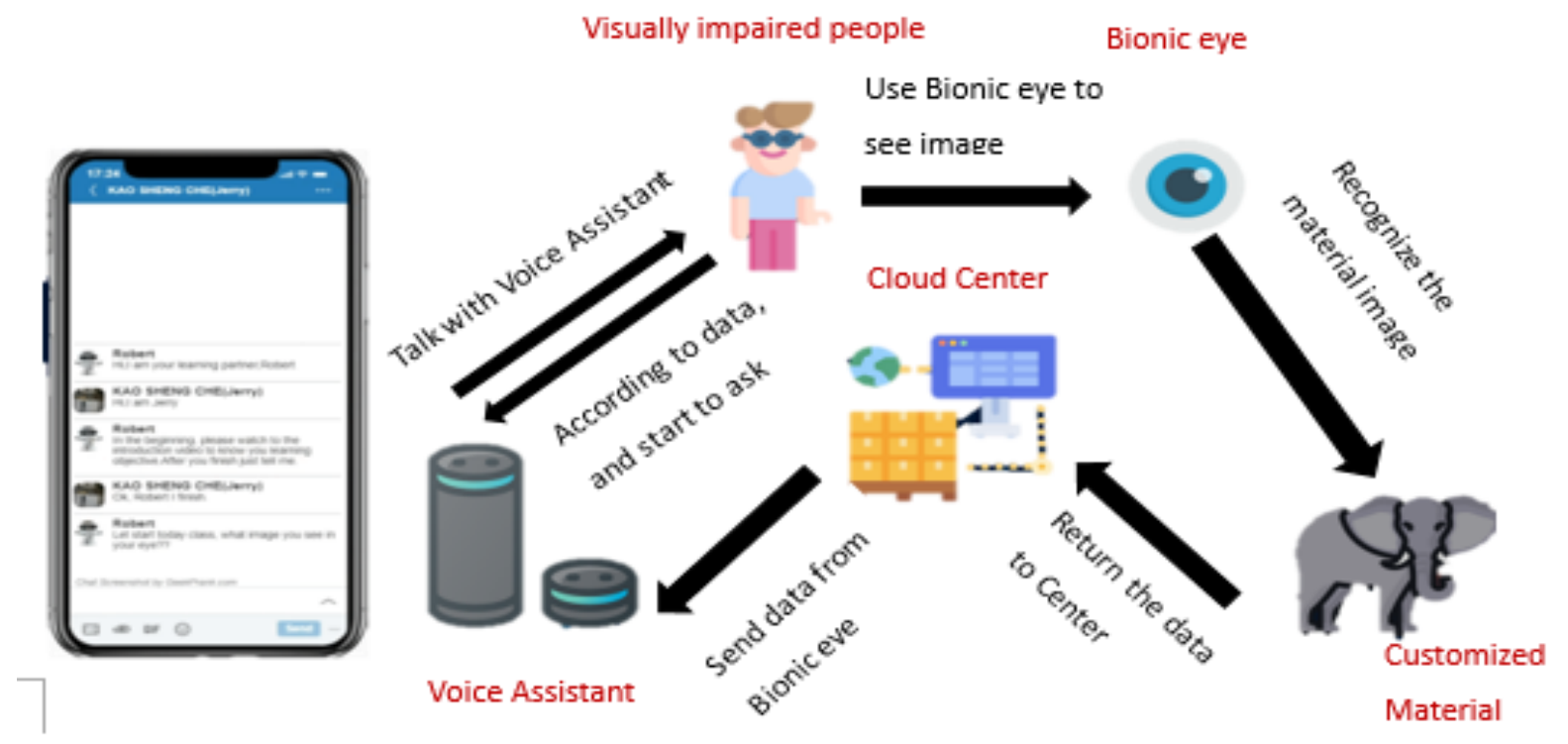

Fig. 5. User Scenario in the system

-Amazon Cognito: Performs user authentication and user management.

-Amazon DymanoDB: Used as additional storage for personal user characteristics required for personalization of learning content.

-Amazon Lex: Directly used for developing chatbot that will perform the interaction learning process and recognize provided data; AWS Lambda, that performs additional analysis of requested information considering personal user characteristics, obtained from DynamoDB, and returns corresponding data to Amazon Lex.

-Amazon CloudWatch: Monitor chatbot operation.

-Amazon S3: Store learning materials, which are tagged in accordance with the level of learner characteristics, valuable for their understanding.

According to the following way we mentioned, we will use the Voice architecture as our development foundation, In the DM (Decision Making) Block, apply the LDA algorithm and COI Framework on this voice assistant original algorithm. And the voice processor will apply on the AWS cloud architecture. As for bionic eye, not only use the component in the article, but also apply it on the AWS cloud architecture. Because both of system all deployed on the cloud service. After the bionic eye get the receiver, it will send it to our cloud database. At the same time, it also trigger the voice assistant's processor to get the data what the blind see now. Then, VA can use history data to interact with the blind student.

\section{DISCUSSION}

This research was focused on finding out the significant effect of voice assistant with bionic eye on visually impaired people. First, we choose the related research which used LDA algorithm. The below chart is from the paper research, After the interaction with the chatbot, 73 students of the last year of the High School responded, according to the Likert scale, to a questionnaire comprising five sections. According to the result, we can assume the LDA can also help voice assistant to chat with students.

\section{Section A: recommendation}

-The proposed services and contents have satisfied the needs of the user, based on personal preferences and the current context.

-The system has furnished effective contents for learning.

\section{Section B: conversation}

-The dialogue with the chatbot took place smoothly and without unexpected jumps

-The system was able to correctly understand the intentions of the user.

\section{Section C: presentation}

-The information has been presented appropriately. 
74 JINOTEP (Jurnal Inovasi dan Teknologi Pembelajaran) Kajian dan Riset dalam Teknologi Pembelajaran Vol. 8, No. 1, Maret 2021, Hal. 68-79

-The information provided was exhaustive

Section D: usability

-The chatbot interface is user-friendly.

-Response times are adequate.

\section{Section E: future developments}

-It would be useful to include in the chat other students.

-It would be interesting to apply the same approach in other scenarios.

Second, We choose the general voice assistant to be our voice teacher, and also adopt its system architecture to combined with our cloud system. The below image is our architecture.
Table 2. CHAT-Context Definition Result from 73 Students (F. Amato et al., 2019)

\begin{tabular}{llll}
\hline Section & Negative & Netural & Postive \\
\hline A & $7,25 \%$ & $4,78 \%$ & $87,97 \%$ \\
B & $8,77 \%$ & $6,41 \%$ & $84,82 \%$ \\
C & $9,10 \%$ & $3,52 \%$ & $87,38 \%$ \\
D & $11,04 \%$ & $3,75 \%$ & $85,21 \%$ \\
E & $7,01 \%$ & $3,15 \%$ & $89,84 \%$ \\
\hline
\end{tabular}

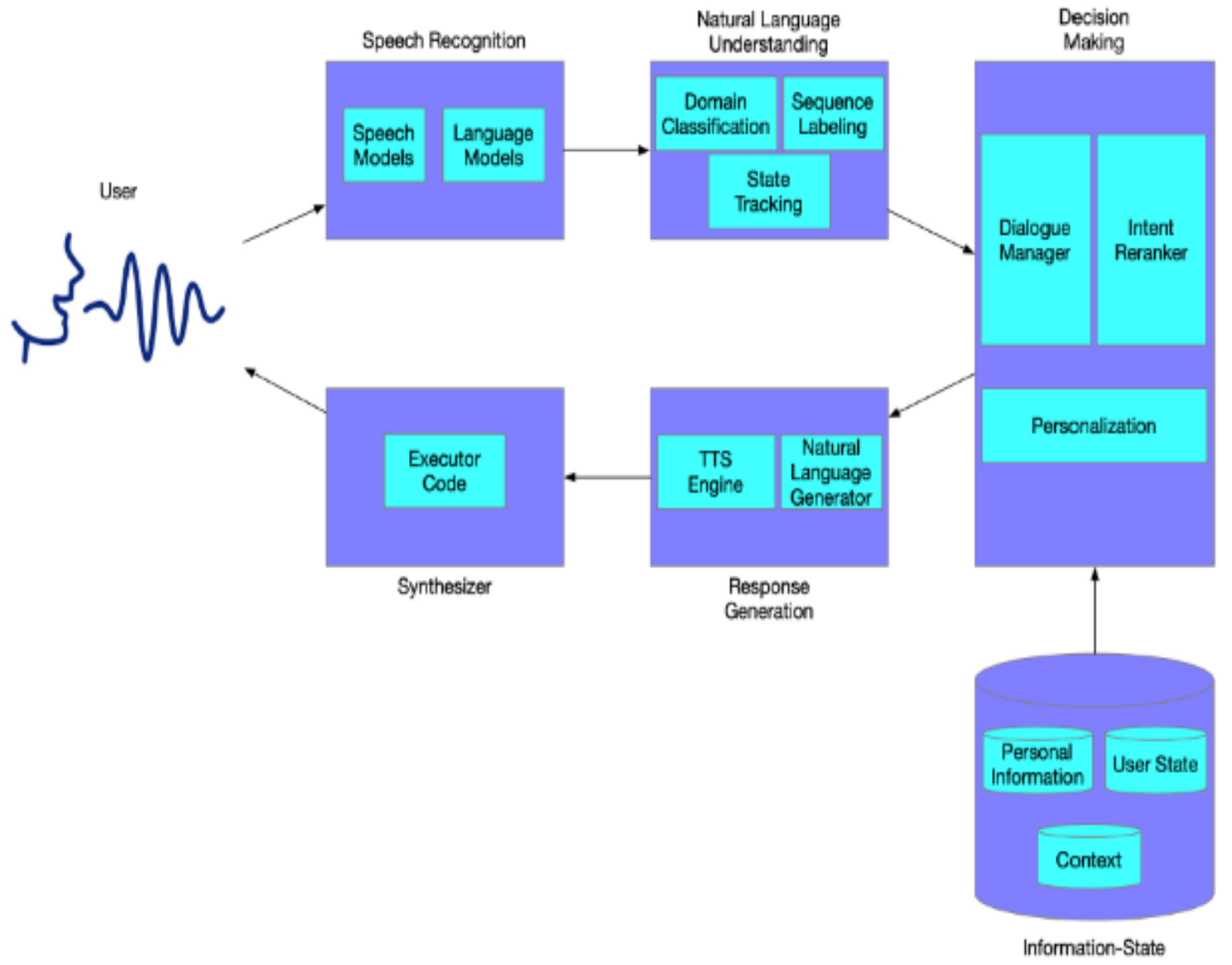

Fig. 6. Voice of Component (R. Anantha et al., 2021)

As for the ASR development in the voice assistant, we want to adopt the More recently, the so-called Bidirectional Encoder Representations from Transformers (BERT) (Devlin, Chang, Lee, \& Toutanova, 2018b) model, a novel neural network-based contextual language model, has shown very impressive results on many natural language processing (NLP). 


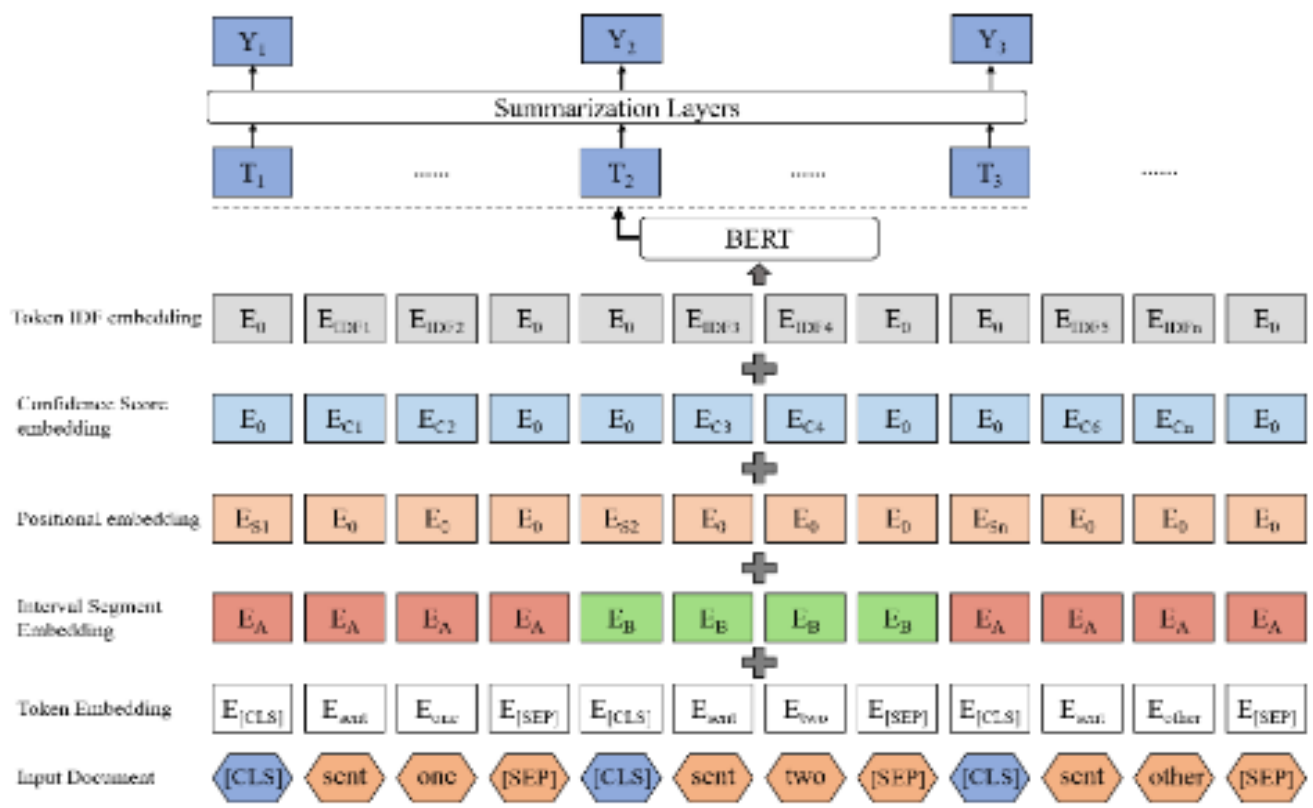

Fig. 7. The architecture of the BERT method (Devlin, Chang, Lee, \& Toutanova, 2018a)

BERT is an innovative neural language model which makes effective use of bidirectional self-attention (also called the Transformer) to capture both short- and longspan contextual interaction between the tokens in its input sequences, usually in the form of words or word pieces (Weng, Lo, \& Chen, 2021). In contrast to the traditional word representation such as word2vec or GLOVE, the advantage of BERT is that it can produce context-aware representation for the same word at different locations by considering bi-directional dependency relations of words across sentences.

BERT has achieved considerable performance gains in almost all types of natural language processing applications, including question answering (QA) (Qu, Qiu, \& Croft, n.d.; Yang, Xie, et al., 2019), information retrieval (IR) (Lin, Lo, \& Chen, 2019; Yang, Zhang, \& Lin, 2019), dialog modeling, and others. Very recently, BERT has made inroads into ex-tractive text summarization for use in identify salient summary sentences (Bert \& Summarization, n.d.) (Lebanoff et al., 2019; H. Zhang, Xu, \& Wang, 2019; Zheng \& Lapata, 2019). According to the result from the paper in different model.
We Finally choose the BERT to be our ASR processor.

Thirdly, because we need bionic eye to support our idea, we also find some research related to bionic eye working (Banerjee et al., 2020).The architecture of bionic eye is below description:

Digital camera: embedded into a pair of glasses. The camera capturing images are done by pupil's movements by monitoring the front of the eye using Infrared illumination. Its main aim is to capture real time images and send to the chip.

Video processing microchip: the images are captured serially and is been processed and convert to electrical pulses which is mostly light and dark and further sends to the transmitter (Paracchini, Marcon, Villa, Zappa, \& Tubaro, 2020).

Transmitter: transmit the pulse to the receiver wirelessly

Receiver: sends the pulses to the retinal implant by a wire which is as thin as a hair

Retinal implant: composed of an array of electrodes on a chip which is about $1 \mathrm{~mm}$ by $1 \mathrm{~mm}$ in size which further triggers the pulse emission. Further the pulse travels through the optic nerves to the brain where vision is being processed and then the brain starts analyzing the 
76 JINOTEP (Jurnal Inovasi dan Teknologi Pembelajaran) Kajian dan Riset dalam Teknologi Pembelajaran Vol. 8, No. 1, Maret 2021, Hal. 68-79

image (Boscain, Prandi, Sacchelli, \& Turco, 2021).

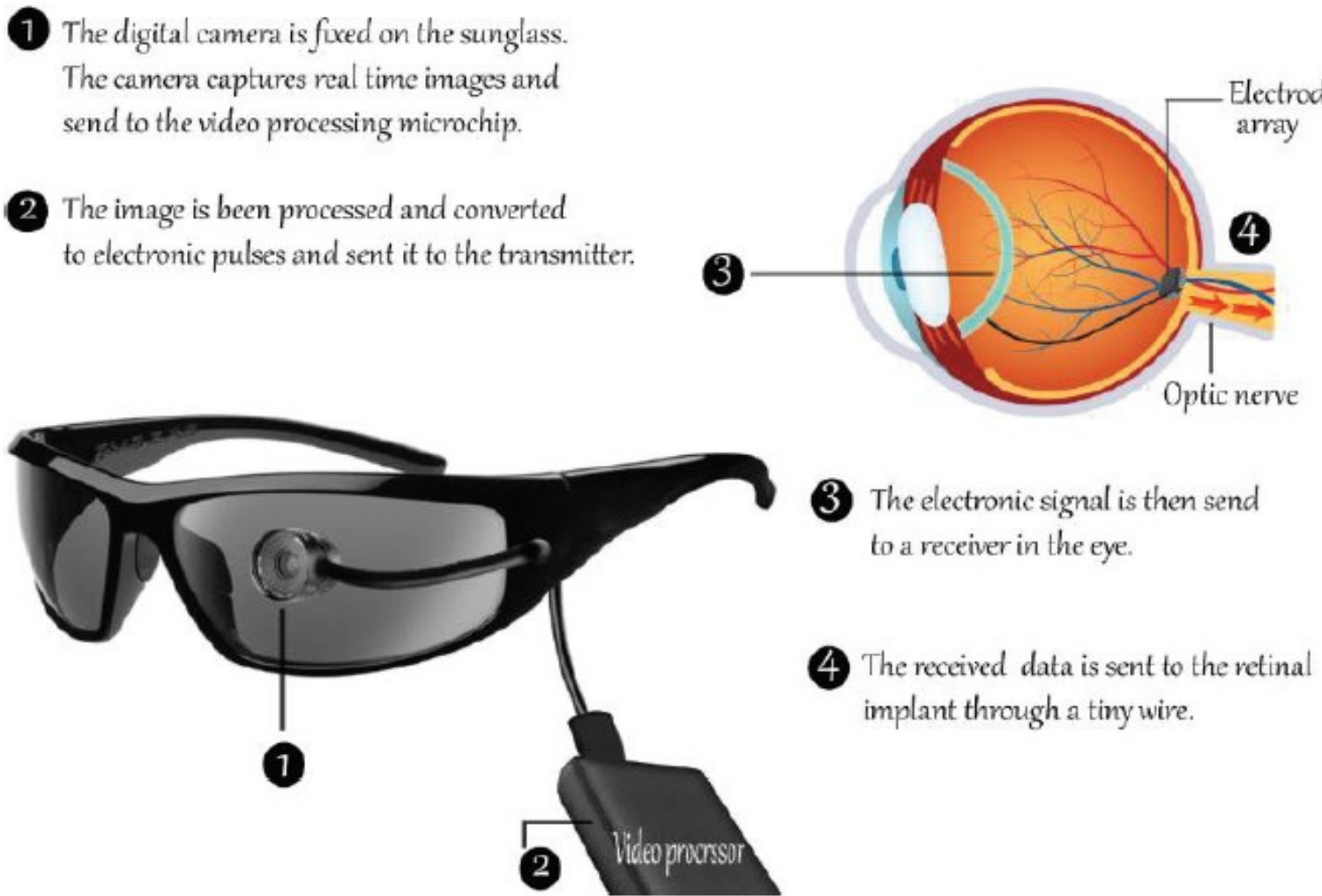

Fig. 8. Working of bionic eye (Banerjee et al., 2020)

Fourthly, because the distance between the electrode and the neuron is a significant factor in determining the current threshold, which is essential for cell activation and has a critical impact on the nature of the obtained signal (McCreery, Yuen, Agnew, \& Bullara, 1997). Advisible material for electrode will be one that will amplify the signal in the extracellular fluid by making electrode-tissue contact more intimate.

To restore the purpose of the nervous system lost because of any diseaseor injury. We choose conductingpolymers (CPs), because it provides excellent electrical conductivityfor signal transduction, along with biocompatibility with human body (Parashar, Prajapati, McIntyre, \& Kandasubramanian, 2020).

Conducting polymers are polymers that conduct electricity because of the presence of conjugate $\pi$-bonds (Masanori Yamada, Goda, Matsukawa, Hata, \& Yasunami, 2015). By doping the $\mathrm{CP}$, the band gap reduces, thus allowing the electrons to transition from one state to other with much less activation energy, making them behave similar to metals. As a result, they have been employed in numerous applications, such as flexible electronics, biosensors (Prajapati \& Kandasubramanian, 2019), hydrophobic functions, stimulated molecular electronics (Magisetty et al., 2020), other electronic applications (Magisetty et al., 2019; Tahalyani, Rahangdale, Aepuru, Kandasubramanian, \& Datar, 2016).

After we discussed a lot of research in this paper including the algorithm, equipment, idea how can we apply it to realized and result from the student satisfactions about the framework. However, there is still need some time integrate these different systems in our cloud database.

Because a team of students have been working with the Interaction Lab at Heriot-Watt University, they recently make some experiment with visually impaired learners, the project called "Conversational Agents and Spoken 
Language Processing" by AyeSaac system to answer with them at home area recently.

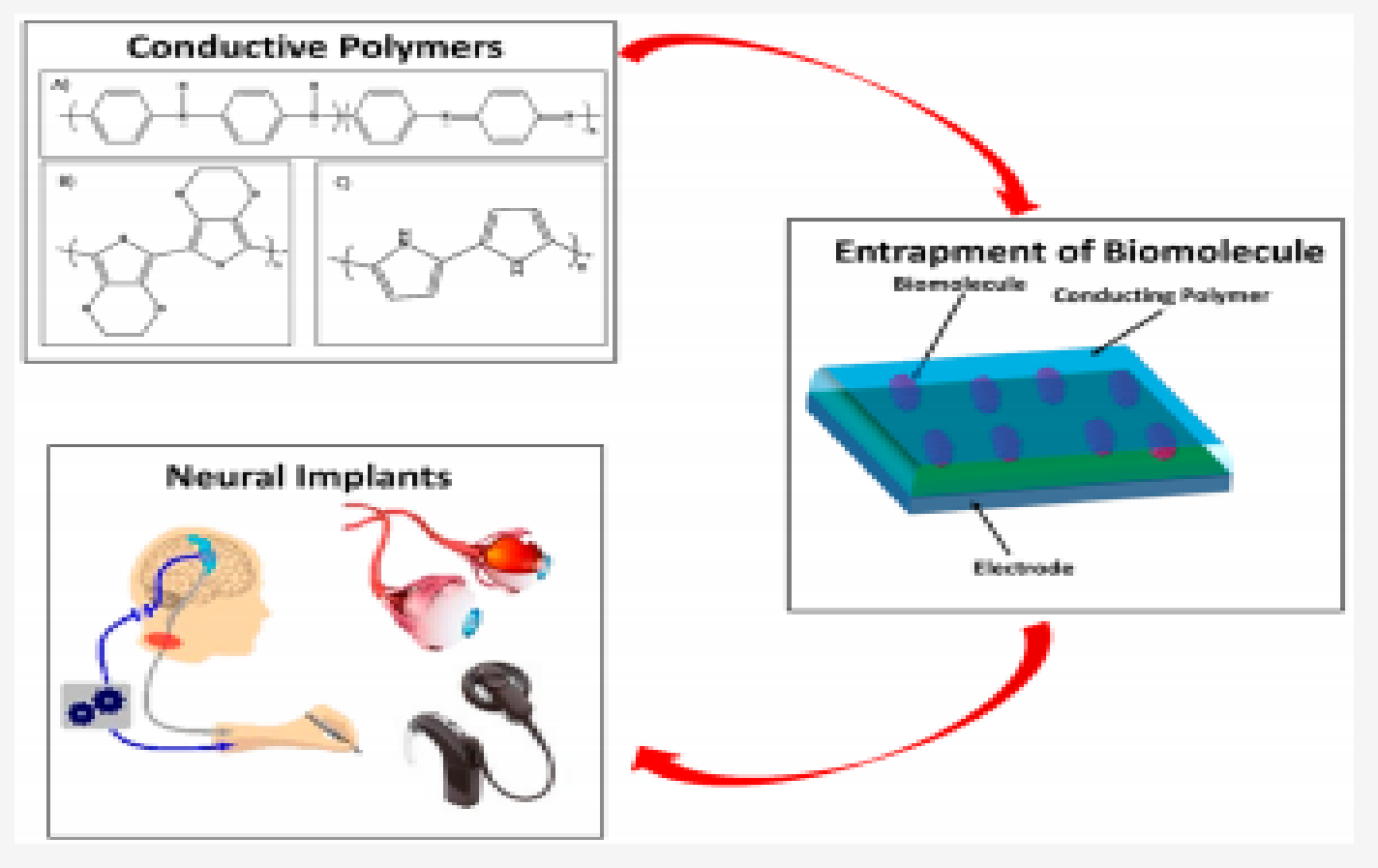

Fig. 10. Conductingpolymers(CPs) applied on the bionic eye system (Parashar et al., 2020)

As for bionic eye, according to the research, Australian University Monash claims that they have invented the first-ever Bionic eye to restore the vision of visually impaired people and will start to test the human experiment. "The design creates a visual pattern from combinations of up to 172 spots of light (Phosphenes) which provides information for the individual to navigate indoor and outdoor environments and recognize the presence of people and objects around them.

Nowadays, these technologies still become advanced and start to be applied in our world, our idea is combined with them together to bring more benefit for the visually impaired learners. They can use the bionic eye to detect the learning material with real teacher of voice assistant. It can make learner have better environment and also improve the learning efficiency.

\section{CONCLUSION}

The main benefit of this idea can really help the visually impaired people learn, Introduction of chatbot into eLearning leads to the appearance of new modification, which is similar with personalized learning. Besides, Voice Assistant are the popular application direction from previous years. VAs could provide customized guidance for instructions dependent on their personal needs. As voice process technology still become better, it's look bright that it can also apply on eLearning in the future. According to the recent great news from bionic eye, it can have identified simple image and start to test on some animals. Such Combination of Voice Assistant and Bionic eye could be a solution to the problem of the lack of image learning for the blinds. This report wants to describes the voice assistant with bionic eye for an eLearning system to the blinds. Integration of this type of voice assistant, we should use cloud architecture, voice processing and learning theory, bionic eye technology to support our idea.

In the future, the next step of our system is to build stable cloud architecture to combined with both of different technology system. And there are still full of obstacles we need to overcome. For example, how can we immediately handle the image processing time and send to the VAs.

In our current version, the application of two technologies cannot totally applied It's still some time to make them become more and more 
78 JINOTEP (Jurnal Inovasi dan Teknologi Pembelajaran) Kajian dan Riset dalam Teknologi Pembelajaran Vol. 8, No. 1, Maret 2021, Hal. 68-79

mature. Hopefully, those technologies will achieve our idea in the future, we can really bring the new world to the blind those who want to learn like us.

\section{REFERENCES}

Amato, F., Casillo, M., Colace, F., Santo, M. D., Lombardi, M., \& Santaniello, D. (2019). CHAT: A Cultural Heritage Adaptive Tutor. TALE 2019 - 2019 IEEE International Conference on Engineering, Technology and Education.

https://doi.org/10.1109/TALE48000.2019.922 5962

Amato, Flora, Casillo, M., Colace, F., De Santo, M., Lombardi, M., \& Santaniello, D. (2019). CHAT: a Cultural Heritage Adaptive Tutor. 2019 IEEE International Conference on Engineering, Technology and Education (TALE), 1-5. IEEE

Anantha, R., Chappidi, S., \& Dawoodi, W. (2021). Learning to Rank Intents in Voice Assistants. In Lecture Notes in Electrical Engineering (Vol. 704). https://doi.org/10.1007/978-98115-8395-7 7

Anantha, Raviteja, Chappidi, S., \& Dawoodi, W. (2020). Learning to Rank Intents in Voice Assistants. In Conversational Dialogue Systems for the Next Decade (pp. 87-101). Springer.

Anderson, T., Liam, R., Garrison, D. R., \& Archer, W. (2001). Assessing teaching presence in a computer conferencing context.

Ayedoun, E., Hayashi, Y., \& Seta, K. (2019). L2 learners' preferences of dialogue agents: A key to achieve adaptive motivational support? International Conference on Artificial Intelligence in Education, 19-23. Springer.

Banerjee, A., Saba, S. B. A., Rana, S., \& Chakraborty, S. (2020). Bionic Eye-A Review. 2020 8th International Conference on Reliability, Infocom Technologies and Optimization (Trends and Future Directions)(ICRITO), 501504. IEEE.

Bert, F., \& Summarization, E. (n.d.). Fine-tune BERT for Extractive Summarization.

Boscain, U., Prandi, D., Sacchelli, L., \& Turco, G. (2021). A bio-inspired geometric model for sound reconstruction. Journal of Mathematical Neuroscience, 11(1). https://doi.org/10.1186/s13408-020-00099-4

Davies, J. N., Verovko, M., Verovko, O., \& Solomakha, I. (2021). Personalization of elearning process using ai-powered chatbot integration. In Advances in Intelligent Systems and Computing. https://doi.org/10.1007/978-3030-58124-4 20

Devlin, J., Chang, M.-W., Lee, K., \& Toutanova, K. (2018a). Bert: Pre-training of deep bidirectional transformers for language understanding. ArXiv Preprint ArXiv: 1810.04805.

Devlin, J., Chang, M. W., Lee, K., \& Toutanova, K. (2018b). BERT: Pre-training of deep bidirectional transformers for language understanding. ArXiv, 4171-4186.

Fryer, L. K., Ainley, M., Thompson, A., Gibson, A., \& Sherlock, Z. (2017). Stimulating and sustaining interest in a language course: An experimental comparison of Chatbot and Human task partners. Computers in Human Behavior, 75, 461-468.

Garcia Brustenga, G., Fuertes Alpiste, M., \& Molas Castells, N. (2018). Briefing paper: los chatbots en educación.

Heller, B., Proctor, M., Mah, D., Jewell, L., \& Cheung, B. (2005). Freudbot: An investigation of chatbot technology in distance education. EdMedia+ Innovate Learning, 3913-3918. Association for the Advancement of Computing in Education (AACE).

Lebanoff, L., Song, K., Dernoncourt, F., Kim, D. S., Kim, S., Chang, W., \& Liu, F. (2019). Scoring sentence singletons and pairs for abstractive summarization. ArXiv Preprint ArXiv: 1906.00077.

Lin, H.-Y., Lo, T.-H., \& Chen, B. (2019). Enhanced BERT-based ranking models for spoken document retrieval. 2019 IEEE Automatic Speech Recognition and Understanding Workshop (ASRU), 601-606. IEEE.

Magisetty, R., Hemanth, N. R., Kumar, P., Shukla, A., Shunmugam, R., \& Kandasubramanian, B. (2020). Multifunctional conjugated 1, 6heptadiynes and its derivatives stimulated molecular electronics: Future moletronics. European Polymer Journal, 124, 109467.

Magisetty, R., Kumar, P., Gore, P. M., Ganivada, M., Shukla, A., Kandasubramanian, B., \& Shunmugam, R. (2019). Electronic properties of Poly (1, 6-heptadiynes) electrospun fibrous non-woven mat. Materials Chemistry and Physics, 223, 343-352.

McCreery, D. B., Yuen, T. G. H., Agnew, W. F., \& Bullara, L. A. (1997). A characterization of the effects on neuronal excitability due to prolonged microstimulation with chronically implanted microelectrodes. IEEE Transactions on Biomedical Engineering, 44(10), 931-939.

Paracchini, M., Marcon, M., Villa, F., Zappa, F., \& Tubaro, S. (2020). Biometric Signals Estimation Using Single Photon Camera and Deep Learning. Sensors, 20(21), 6102.

Parashar, K., Prajapati, D., McIntyre, R., \& Kandasubramanian, B. (2020). Advancements in biological neural interfaces using conducting polymers: a review. Industrial \& Engineering Chemistry Research, 59(21), 9707-9718. 
Prajapati, D. G., \& Kandasubramanian, B. (2019). Progress in the development of intrinsically conducting polymer composites as biosensors. Macromolecular Chemistry and Physics, 220(10), 1800561.

Qu, C., Qiu, M., \& Croft, W. B. (n.d.). BERT with History Answer Embedding for Conversational Question Answering.

Ruan, S., Jiang, L., Xu, J., Tham, B. J.-K., Qiu, Z., Zhu, Y., ... Landay, J. A. (2019). Quizbot: A dialogue-based adaptive learning system for factual knowledge. Proceedings of the 2019 CHI Conference on Human Factors in Computing Systems, 1-13.

Schilling, M. A., \& Shankar, R. (2019). Strategic management of technological innovation. McGraw-Hill Education.

Srivastava, S., Kalra, S., \& Prabhakar, T. V. (2020). Contextual Reactive Pattern on Chatbot building Platforms. Proceedings of the European Conference on Pattern Languages of Programs 2020, 1-8.

Srivastava, S., \& Prabhakar, T. V. (2020). Desirable Features of a Chatbot-building Platform. 2020 IEEE International Conference on Humanized Computing and Communication with Artificial Intelligence (HCCAI), 61-64. IEEE.

Tahalyani, J., Rahangdale, K. K., Aepuru, R., Kandasubramanian, B., \& Datar, S. (2016). Dielectric investigation of a conducting fibrous nonwoven porous mat fabricated by a one-step facile electrospinning process. RSC Advances, 6(43), 36588-36598.

Torres-Signes, A., \& Dip, J. A. (2020). A Bayesian Functional Methodology for Dengue Risk Mapping in Latin America and the Caribbean. Acta Tropica, 105788.

Weber, K., Ritschel, H., Lingenfelser, F., \& André, E. (2018). Real-Time Adaptation of a Robotic Joke Teller Based on Human Social Signals. Proceedings of the 17th International
Conference on Autonomous Agents and MultiAgent Systems, 2259-2261. International Foundation for Autonomous Agents and Multiagent Systems.

Weng, S.-Y., Lo, T.-H., \& Chen, B. (2021). An Effective Contextual Language Modeling Framework for Speech Summarization with Augmented Features. 2020 28th European Signal Processing Conference (EUSIPCO), 316-320. IEEE.

Yamada, M., Goda, Y., Matsukawa, H., Hata, K., \& Yasunami, S. (2016). A Computer-Supported Collaborative Learning Design for Quality Interaction. IEEE Multimedia, 23(1), 48-59. https://doi.org/10.1109/MMUL.2015.95

Yamada, Masanori, Goda, Y., Matsukawa, H., Hata, K., \& Yasunami, S. (2015). A computersupported collaborative learning design for quality interaction. IEEE MultiMedia, 23(1), 48-59.

Yang, W., Xie, Y., Lin, A., Li, X., Tan, L., Xiong, K., ... Lin, J. (2019). End-to-end open-domain question answering with bertserini. ArXiv Preprint ArXiv: 1902.01718.

Yang, W., Zhang, H., \& Lin, J. (2019). Simple applications of BERT for ad hoc document retrieval. ArXiv Preprint ArXiv: 1903.10972.

Zhang, H., Xu, J., \& Wang, J. (2019). Pretrainingbased natural language generation for text summarization. ArXiv Preprint ArXiv:1902.09243.

Zhang, Q., He, Y.-J., Zhu, Y.-H., Dai, M.-C., Pan, M.-M., Wu, J.-Q., ... Xu, X.-R. (2020). The evaluation of online course of Traditional Chinese Medicine for MBBS international students during the COVID-19 epidemic period. Integrative Medicine Research, 100449.

Zheng, H., \& Lapata, M. (2019). Sentence centrality revisited for unsupervised summarization. ArXiv Preprint ArXiv: 1906.03508. 